\title{
Cancer drivers and clonal dynamics in acute lymphoblastic leukaemia subtypes
}

\author{
James B. Studd $\mathbb{D}^{1 凶}$, Alex J. Cornish (D) ${ }^{1}$, Phuc H. Hoang $\mathbb{D}^{1,2}$, Philip Law $\mathbb{D}^{1}$, Ben Kinnersley (D) ${ }^{1}$ and Richard Houlston (D) ${ }^{1}$
}

(c) The Author(s) 2021

To obtain a comprehensive picture of composite genetic driver events and clonal dynamics in subtypes of paediatric acute lymphoblastic leukaemia (ALL) we analysed tumour-normal whole genome sequencing and expression data from 361 newly diagnosed patients. We report the identification of both structural drivers, as well as recurrent non-coding variation in promoters. Additionally we found the transcriptional profile of histone gene cluster 1 and CTCF altered tumours shared hallmarks of hyperdiploid ALL suggesting a 'hyperdiploid like' subtype. ALL subtypes are driven by distinct mutational processes with AID mutagenesis being confined to ETV6-RUNX1 tumours. Subclonality is a ubiquitous feature of ALL, consistent with Darwinian evolution driving selection and expansion of tumours. Driver mutations in B-cell developmental genes (IKZF1, PAX5, ZEB2) tend to be clonal and RAS/RTK mutations subclonal. In addition to identifying new avenues for therapeutic exploitation, this analysis highlights that targeted therapies should take into account composite mutational profile and clonality.

Blood Cancer Journal (2021)11:177; https://doi.org/10.1038/s41408-021-00570-9

\section{INTRODUCTION}

Acute lymphoblastic leukaemia (ALL) is the most common childhood cancer, with around $80 \%$ of ALL cases derived from B-cell precursors (BCP-ALL) [1]. The disease is characterised by initiating genetic lesions resulting in characteristic patterns of chromosomal gain (hyperdiploidy), loss (hypodiploidy) or the formation of fusion genes. Recurrent fusions include t(12;21) ETV6RUNX1, $\mathrm{t}(1 ; 19)$ TCF3-PBX1 and $\mathrm{t}(9 ; 22) B C R-A B L 1$ [1]. Copy number changes in $R U N X 1$, caused by intra-chromosomal amplification (iAMP21), and ERG deletion, have also more recently been recognised as initiating events $[2,3]$. The biological differences between subtypes is reflected in their clinical behaviour [4-7]. For example patients with hyperdiploid ALL have 5-year survival rate (5YSR) of $>90 \%$ [7]. In comparison around $60 \%$ of iAMP21 positive ALL will relapse resulting in 5YSR of only 29\% [5].

Current first-line therapy for ALL is dominated by chemotherapeutic and steroidal agents. While their use has driven 5YSR to $>90 \%$ [8], this is at the expense of significant morbidity, and despite these improvements, survival for relapsed ALL is only 21-39\% $[9,10]$. Strategies for developing novel therapies for ALL have largely focused on monoclonal antibodies or CAR-T cells. Such therapies are expensive; when licensed, the anti-cd19 monoclonal blinatumomab, was the most expensive cancer therapy ever [11]. It is therefore desirable to develop additional targeted small molecule therapies to reduce treatment-associated morbidity and relapse-associated cost. Such developments are likely to require precise molecular characterisation and risk stratification, informed by our understanding of ALL genomics.

Precancerous lesions harbouring initiating events can be undetected for years usually requiring the acquisition of additional genetic lesions for symptomatic disease. Common secondary lesions impact genes regulating the cell cycle (CDKN2A, RB1), B-cell development (PAX5, IKZF1, EBF1) and the RAS/RTK pathway (NRAS, $K R A S, F T L 1)$ [1]. Deletions of both CDKN2A and PAX5 occur in around $30 \%$ of tumours. Activation of RAS-RTK genes while most common in hyperdiploid ALL, is observed in $35 \%$ of all tumours [12]. While the landscape of coding mutations in BCP-ALL has been well characterised [13-17], the full complement of molecular lesions sufficient to cause ALL, and explain its biological diversity are unknown.

To obtain a more comprehensive picture of the composite genetic events acting in concert in each of the BCP-ALL subtypes, we performed a genomic analysis of diagnostic samples from 361 ALL patients (Supplementary Fig. 1). We identify noncoding and copy number drivers. Our analysis also reveals differences in the mutational and biological pathways influencing the initiation and progression of disease subtypes.

\section{METHODS \\ Cases, data and sequencing}

Matched tumour-normal whole genome sequencing (WGS) data from 361 treatment-naïve cases of paediatric $(<18$ years old) BCP-ALL were obtained from St. Jude Research Hospital (https://www.stjude.cloud/). Data were accessed and analysed through the DNAnexus cloud computing platform. Ethical permission was not required as all data were in the public domain.

WGS data were generated using $100 \mathrm{bp}$ paired-end libraries (average read depth $45 \times$ and $62 \times$ for normal and tumour samples respectively), using Illumina (San Diego, USA) HiSeq2000 technology. Raw sequencing data were aligned with BWAmem v0.7.17 [18] to GRCh38, by Google Genomics. Cross-contamination was assessed using GATK v4.0.0.1; no sample having $>2.6 \%$. Tumour RNA sequencing (RNA-seq) on 222 (post quality control [QC]) of the 361 cases was performed on 125 bp paired-end

${ }^{1}$ Division of Genetics and Epidemiology, The Institute of Cancer Research, Sutton SM2 5NG, UK. ${ }^{2}$ Present address: Division of Cancer Epidemiology and Genetics, National Cancer Institute, Bethesda, ML, USA. ${ }^{\bowtie}$ email: james.studd@icr.ac.uk 
libraries using Illumina HiSeq technology to an average number of $55 \times 10^{6}$ reads. RNAseq fastqs were analysed using FastQC and aligned to GRCh38 using STAR v2.6.1 [19], discarding samples with $<20 \%$ of reads aligning to the genome. Transcript abundance calculated in transcripts per million (TPM) using RSEM v1.3.0 [20] using GENCODEv30 annotation and was adjusted for batch effects using ComBat-seq [21].

Fusion genes were identified from RNAseq data using STAR-Fusion v1.5.018 and FusionInspector [22]. Candidate fusions were retained when fusion genes were separated by $>1 \mathrm{MB}$ and absent from control samples. For controls, we used unmatched lymphoblastoid cell line data (GTEx 1000 Genomes RNA sequencing project [23] [ $n=465]$ ) and unmatched mixed tissue samples (Human Protein Atlas Project [24] $[n=200]$ ), processed using the same pipeline as tumour samples. Links provided in the web resources section.

The transcriptional impact of histone gene cluster 1 (chr6:2612268526239852) deletion and CTCF alteration (deletion or mutation) were assessed using DESeq2 v1.329 [25], with default settings. Tumours were divided into three groups; two test groups possessing an alteration in CTCF or the histone gene cluster 1, excluding those with alterations in both and a control group. Test groups comprised those with gene(s) deletion ( $<2$ copies), of which 17 tumours had associated RNAseq data (CTCF altered $n=9$; histone 1 cluster altered $n=8$ ).

\section{Variant calling}

Somatic single nucleotide variants (SNVs) and indels were called in the 361 matched tumour-normal pairs using Strelka v2.8.4 [26] in tumour mode, adopting default parameters. QC filtering of somatic variants comprised: (1) Retaining only variants marked as 'PASS'; (2) Excluding variants seen in the panel of 160 matched germline samples (generated by running Strelka2 in germline mode); (3) Excluding variants in repetitive regions (extracted from UCSC) or in homopolymer runs of $>7$ nucleotides; (4) Excluding variants with a POPMAX allele frequency $>0.001$ in GnomAD v3, (5) Excluding variants with a $\mathrm{VAF}<5 \%$. Driver mutation plot generated using Maftools [27].

\section{Mutational signatures}

De novo extraction of signatures was performed using SigProfilerExtractor v1.0.18 [28]. Extracted signatures were assigned to reference signatures from Catalogue of Somatic Mutations in Cancer (COSMIC) v3.1 using a minimum cosine similarity threshold of 0.9 .

\section{Identification of cancer drivers and pathways}

Identification of SNV/indel drivers in coding regions was based on a consensus-based approach. Per gene $P$ values were calculated combining the output of MutSigCV2 v3.11 [29], dndsCV v0.0.1 [30] and OncodriveFML [31] using Harmonic means [32] and Benjamini-Hochberg correction. Variants were classified as nonsilent using variant effect annotator (VEP) [33] annotations (Supplementary Table 1). Candidate drivers were filtered retaining genes: (1) significant $(P<0.05)$ in $\geq 2$ methods, (2) corresponding RNAseq expression (mean $>0.02$ TPM) and (3) mutated in $\geq 5$ tumours.

To identify driver mutations in enhancer regions we adopted the strategy of Orlando et al. [34]. Cis-regulatory elements (CREs) were identified from promoter capture chromatin confirmation ( $\mathrm{PHi}-\mathrm{C}$ ) contacts (CHiCAGO score > 5) from naïve B-cells [35]. CRE-specific mutation probabilities, for each tumour, were generated using logistic regression preformed with the $R$ package $\mathrm{glm}$, accounting for base composition, mutation rate, replication timing, and coverage. Replication timing was extracted for the lymphoblastoid cell lines (GM12878, GM12813, GM12812, GM12801, GM06990), link provided in the web resources section. The Poibin $R$ package was used for approximation of Poisson binomials, deriving empiric $P$ values regions as per Melton et al. [36].

CREs harbouring $>5$ mutations were examined for mutational clustering by permutation $(n=10,000)$ assuming uniform mutation distribution, deriving empiric $P$ values. Frequency and clustering $P$ values were combined using Fisher's method and adjusted for multiple testing using Benjamini-Hochberg correction. Genome regions with a $Q$ value $<0.1$ were examined for transcriptional effects. Expression of genes were captured by an interaction were compared between mutated and nonmutated samples using Benjamini-Hochberg corrected Wilcox rank-sum test, excluding tumours with CNVs at either the target gene or CRE.

Mutation burden in promoters and UTR regions was assessed using OncodriveFML [31]. Promoters (defined from the transcription start site -2KB) were extracted from GENCODEv30 GRCh38.p12. Where genes had multiple transcription start sites all promoter sequences were evaluated jointly. Promoters were filtered for any overlapping coding or UTR sequence.

For pathway analysis driver genes were manually assigned to biological pathways. Gene-pathway assignments are described in Supplementary Table 2. To calculate the overrepresentation of alterations in biological pathways we compared the alteration frequency of each subtype to the remaining subtypes.

\section{Identification of copy number and structural variants}

Somatic copy number variation (CNV) was called using CNVkit v0.9.5.3 [37]. Tumour WGS data were called against a pooled reference, generated from 45 representative matched germline samples ( 23 male, 22 female). CNVkit segment specific coverage log2 ratios were adjusted for tumour cell purity, estimated by cpgBattenberg [38]. Copy number states were assigned using default log2 thresholds $(<-1.1=0,>-1.1=1,>0.4=2$, $>0.3=3,>0.7=4)$. CNVs were considered 'arm' level when an alteration occupied $>80 \%$ of mappable chromosome arm length. Other variants were defined as 'focal'. Additional copy number assessments were made using HMMcopy v1.32 calculating GC and mappability normalised tumour/normal log2 coverage ratios.

Driver CNVs were called using GISTIC2 v2.0.23 [39] run in focal mode (excluding arm level events) with default parameters. Genome regions were excluded if they: (1) overlapped a immunoglobulin locus, (2) contained no protein coding genes, (3) contained no genes expressed in corresponding RNAseq data (excluding deleted cases), (4) the region was significantly amplified and deleted, (5) Q-value $>0.01$.

Structural variants (SVs) were called using Manta v1.5 [40], Lumpy v0.2.13 [41] and Delly2 v0.8.1 [42]. Manta and Delly2 were run using default parameters. Lumpy was run using the wrapper Smoove v0.2.3. Variants were excluded if they were located in centromeric, telomeric or heterochromatic regions, had a variant allele frequency $(\mathrm{VAF})<0.1$, or occurred in a panel of matched normals, generated using the corresponding method. The remaining variants were merged as per Li et al. [43], retaining only those called by $\geq 2$ methods.

SV cancer cell fractions (CCFs) were estimated using SVclone [44] and SV clustering examined using ClusterSV [43]. Regions of chromothripsis were identified using ShatterSeek v0.4 [45], based on thresholds of $>3$ adjacent segments of oscillating copy number involving $>5$ interleaved SVs. Candidate chromothripsis events were manually reviewed.

To jointly analyse CNVs and SVs, regions called by GISTIC were additionally filtered, retaining those enriched in overlapping SVs. For CNV regions, corresponding (deleted/amplified) simple SVs (not part of complex rearrangements called by SVClust) were examined. Chromosomearm-specific background SV rates were estimated by permutation $(n=$ 1000). $P$ values were computed as the proportion of permutations where the number of simulated SVs overlapping a locus was greater than or equal to the number of observed SVs. Regions enriched $(P<0.01)$ in overlapping SVs were retained.

SV breakpoint motif enrichment was performed using HOMER v4.10.4 [46], by extracting two $100 \mathrm{bp}$ sequences ( $\pm 50 \mathrm{bp}$ ) from each breakpoint, excluding SVs where both breakpoints mapped to immunoglobulin regions (Supplementary Table 3). HOMER annotates motifs with the most similar sequence, based on Pearson $r^{2}$, from the JASPAR [47] database. Annotated HOMER motifs were further processed with reference to motifs of candidate mutagenic drivers of ALL (Supplementary Table 4). Where the Pearson correlation between a HOMER motif and a candidate mutagenic motif exceeded the most similar JASPAR annotation they were substituted. Motifs with a correlation of $<0.85$ were excluded.

\section{Tumour subtyping}

Tumour chromosomal ploidy was based on copy number data from CNVkit. Tumours with a chromosome number $>50$ were classified as hyperdiploid and those with $<45$, hypodiploid. Near haploid tumours $(\mathrm{n}=$ 11) chromosome number $24-30$ are included the hypodiploid subtype unless otherwise stated. iAMP21 status was called as per Harrison et al. [48]. Chromosomal ploidy was also called using clonal copy number calls from cpgBattenberg $(n=280)$. For samples with divergent chromosome numbers a manual review was performed, resulting in 3 samples being reclassified from hyperdiploid to near haploid.

Subtypes defined by fusion events (e.g., ETV6-RUNX1) were assigned based on clonal SVs consistent with fusion gene expression and corresponding fusion RNAseq expression. Cases without an established 

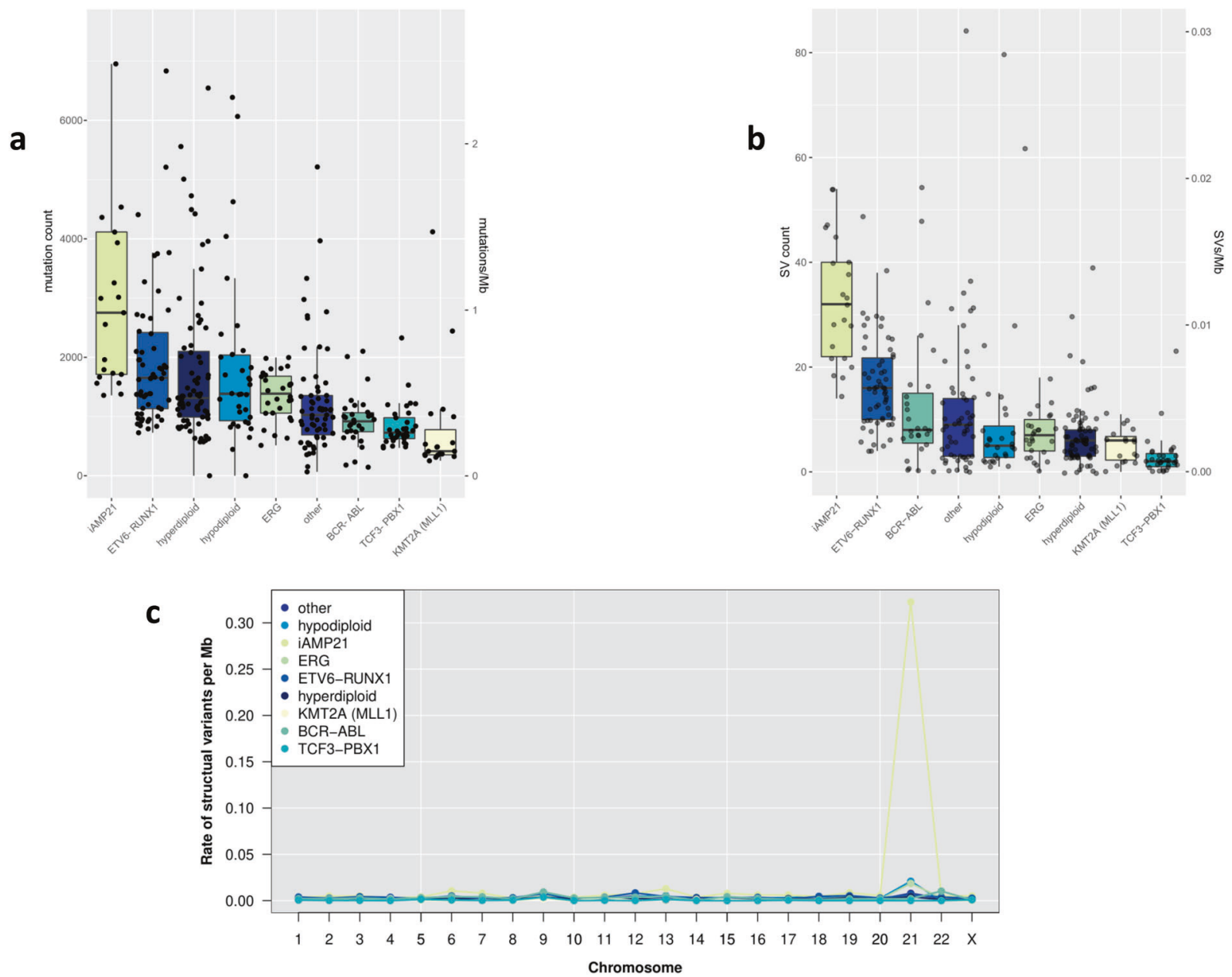

Fig. 1 Mutation burden by subtype. Short somatic variants (SNVs and indels) were called in 361 matched normal/tumour whole genome sequencing samples. a Burden of SNVs and indels. Box and whiskers plot of mutation count per tumour. b Burden of structural variants (SVs). Box and whiskers plot of SV count, dots represent individual tumours. c Plot of the SV rate per chromosome retaining only intrachromsomal variants outside immunoglobulin loci. $Y$-axis; mean SVs rate per Mb. $X$ - axis; chromosome.

initiating driver event were designated as unclassified/other. The subtype composition of the cohort is detailed in Supplementary Fig. 2.

\section{Clonality and tumour evolution}

Tumour ploidy and SNV cancer cell fractions (CCF) were estimated using cpgBattenberg v3.5.0 [38], adopting default parameters except minimum ploidy was thresholded at 1.1. Single nucleotide polymorphisms alleles from the 1000 Genomes Project (v3, GRCh38) were counted in tumour and normal samples, and genotypes phased using impute2 [49]. Puritycorrected copy number segments were used to compute SNV/indel CCFs and subclones identified by DPClust v2.28 [38], assigning somatic variants to clusters. Clusters with the highest CCF $>0.9$ and $<1.1$ were considered clonal. Samples were excluded based on the following criteria: (1) a variant cluster with CCF > 1.1; (2) no clonal cluster (CCF 0.9-1.1); (3) copy number state-specific SNVs which failed to cluster at predicted VAFs; (4) copy number solutions with homozygous deletions $>3 \mathrm{Mb}$. In the first instance, samples were analysed using Battenberg derived purity estimates, when resulting copy number solutions failed QC CCube v1.0 [50] estimates were used. 280 samples satisfying QC criteria were retained. Tumour cell purity estimates are detailed in Supplementary Fig. 3. To calculate driver gene mutation burden in clonal and subclonal compartments we used cluster assignments from DPClust. The frequency of clusters containing $\geq 1$ damaging driver (Supplementary Table 5) mutations were calculated for each subtype and compared to the remaining subtypes. Heterogeneity was estimated using the Simpson Index (probability that two individuals/ cells, selected from a population/tumour, are from the same species/ clone), calculated using VEGAN [51]. Evidence to support neutral evolution was sought using MOBSTER v0.1.1 [52], as per authors recommendations (retaining only SNVs and indels in diploid regions). MOBSTER identifies variants with a VAF distribution consistent with neutral evolutionary processes, termed a "neutral tail". Variants belonging to neutral, subclonal or clonal clusters were analysed using dNdSCV. The mutation rate of ALL drivers in neutral, subclonal or clonal clusters was calculated as the number of nonsynonymous variants/all non-synonymous sites/total number of mutations (Supplementary Table 5).

\section{RESULTS}

\section{Mutation burden}

As previously documented, the burden of SNVs and indels was low (median $0.43 \mathrm{Mb}^{-1}$, range $0.023-5.29$ ) when compared to the majority of solid cancers. Mutation burdens differed significantly across subtypes $\left(P_{\text {Kruskal-Wallis }}=2.2 \times 10^{-16}\right)$, iAMP21 and KMT2A (MLL1) positive tumours having the highest and lowest burdens respectively (Fig. 1a). The most common chromosome-arm level aberrations were loss of $9 p$ (containing CDKN2A/CDKN2B) and gain of $21 \mathrm{q}$ (containing RUNX1), both occurring in $8 \%$ of cases (Supplementary Fig. 4). 21q gain occurred preferentially in hypodiploid tumours $(Q=0.10)$, whereas $9 p$ loss was most frequent in TCF3-PBX1 translocated tumours $(Q=0.19)$, and (Supplementary Fig. 5a and 5b).

The median number of SVs was eight per tumour $\left(2 \times 10^{-3} \mathrm{Mb}^{-1}\right)$, iAMP21 tumours possessing the highest number (Fig. 1b). The genome-wide distribution of SVs is shown in Supplementary Fig. 6. 


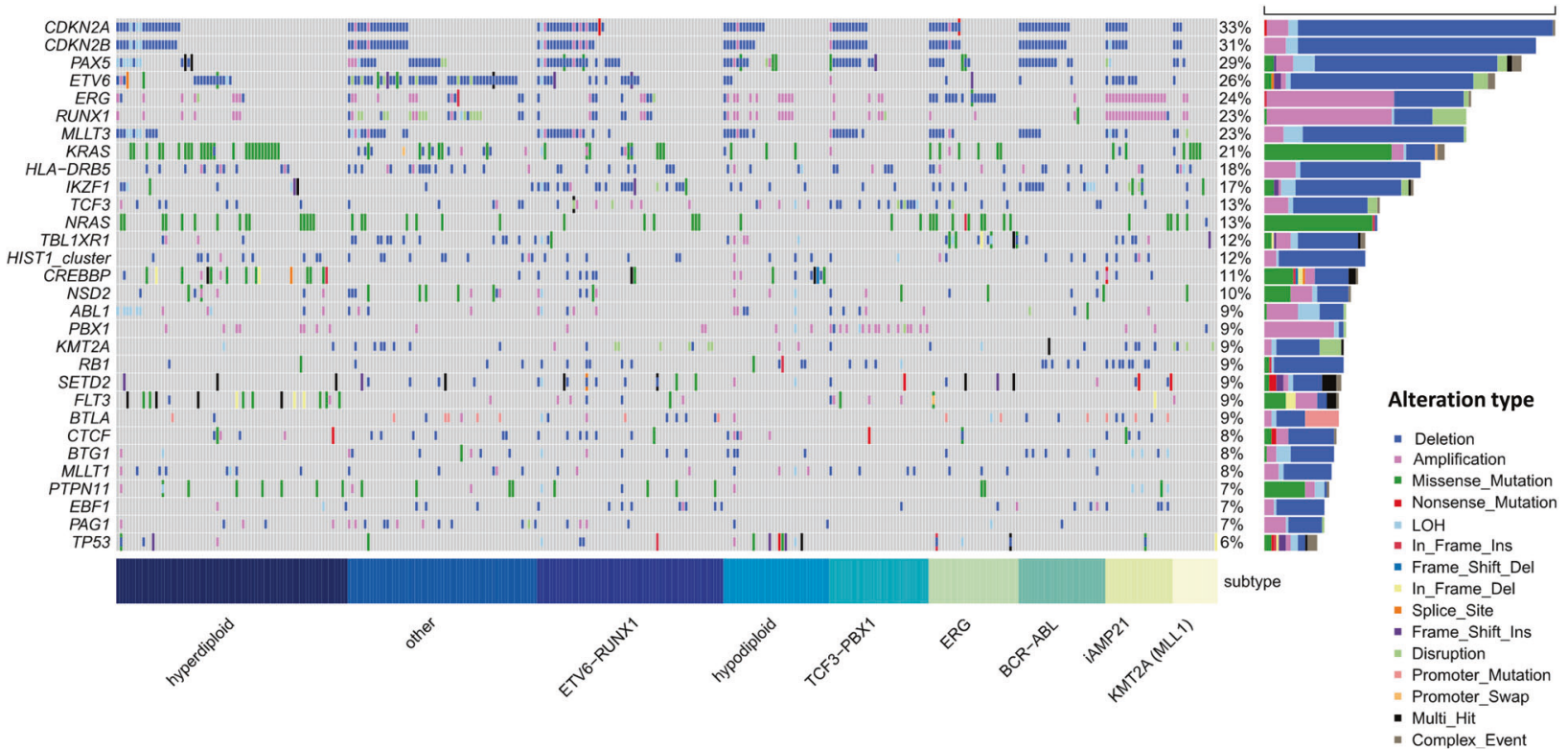

Fig. 2 Driver gene analysis. Oncoplot of somatic alterations for selected ALL driver genes (Supplementary Table 10), genes altered in $>20$ tumours, compiled from SNV, indel, CNV (only focal events), SV, RNAseq, and LOH. Vertical lines represent one tumour. Coloured sections in grey grid denote alteration type, described in key - "Alteration type". Short nucleotide variants span the entire row, other alterations span half the row width. The right bar plot shows the frequency of driver gene alteration, colour denotes alteration type, as prior. Deletions and amplifications derived from CNVs and SVs; disruption from RNAseq and SVs. Alterations are shown non-redundantly; tumours with multiple alterations in the same gene only counted once. Plot generated using Maftools [6] after the exclusion of genes in the psuedoautosomal regions.

Chromosome $21 \mathrm{SV}$ s rates were 10 -fold higher $\left(0.027 \mathrm{Mb}^{-1}\right)$ than other chromosomes, largely accounted for by iAMP21 tumours (Fig. 1c). Since iAMP21 tumours are defined by RUNX1 copy number, we examined the distribution of SVs on chromosome 21, finding no clustering evident (Supplementary Fig. 7). Chromothripsis did not account for elevated SV rates in iAMP21 tumours as no events were observed on chromosome 21.

\section{Identification of driver genes}

We searched for drivers of ALL by first considering the following classes of somatic coding alterations; single nucleotide variants (SNVs)/indels, copy number variants (CNVs), structural variants (SVs) and loss of heterozygosity (LOH). In addition to established drivers, we identified a number of candidate novel ALL drivers, including HLA-DRB5, the histone gene cluster 1, USP8 and CHID1.

Consistent with previous reports [1, 53], the most frequently altered genes included CDKN2A/B, PAX5, ETV6, ERG, RUNX1, NRAS, KRAS and IKZF1 (Fig. 2). By jointly analysing CNV and SV data, we identified two novel regions of recurrent alteration. Firstly, a $120 \mathrm{~kb}$ region of $H L A(6 \mathrm{p} 21 ; 32,442,465-32,554,750 \mathrm{bps})$ was deleted in $17 \%$ of tumours (Fig. 3a). Within this region only $H L A-D R B 5$ was expressed and deletion was associated with significantly reduced gene expression $\left(P_{\text {Mann-Whitney }}=3.7 \times 10^{-4}\right)$. We further evaluated read depth data in tumours with an HLA-DRB5 SVs but lacking a CNV using an additional copy segmentation algorithm [54], finding evidence of a corresponding change number change within $2,000 \mathrm{bp}$ an SV breakpoints in every tumour (Supplementary Fig. 8). Secondly, $117 \mathrm{~kb}$ of $6 \mathrm{p} 22.2$ overlapping histone gene cluster 1 $(26,122,685-26,239,852 \mathrm{bps})$ was deleted in $10 \%$ of tumours (Fig. 3b), deletion was associated with reduced expression of HIST1H4E $\left(P_{\text {Mann-Whitney }}=0.034\right)$ and HIST1H2AE $\left(P_{\text {Mann-Whitney }}=\right.$ 0.023). We estimated SV cancer cell fraction (CCF) using SVclone [44] finding the majority of these variants in the region are clonal.

We observed nonsilent SNVs or indels in USP8, BSN and SLC35G5 in $1.9,1.7$ and $1.4 \%$ of tumours respectively (Supplementary Tables 6,7 and Supplementary Fig. 9). USP8 missense mutations were clustered at three base positions, consistent with oncogenic activation (Supplementary Fig. 10). While the frequency of USP8 variants in the gNOMAD v3 database was $<0.001$, further curation revealed each variant occurred above frequency filter thresholds in legacy releases of the ExAC database indicating they may be technical artefacts. None of the variants in $B S N$ were recurrent and all predicted to be damaging by SIFT and PolyPhen (Supplementary Table 7). Variants in SLC35G5 were predicted to be benign and occurred in ExAC inconsistent with driver function.

Next, we sought to identify non-coding driver mutations. We observed a significant excess of promoter mutations for BTLA (4.2\%, $Q=0.002)$ and CHID1 (2.2\%, Q =0.049). BTLA promoter mutations clustered within a $27 \mathrm{bp}$ region, and were associated with 5 -fold reduced BTLA expression $\left(P_{\text {Mann-whitney }}=0.056\right)$, the small number of tumours with expression data presumably preventing this relationship attaining significance (Fig. 4a). By analysing transcription factors (TFs) with evidence of BTLA promoter binding in ChIPseq, we found each variant tumour possessed a mutation predicted to disrupt TF binding, most frequently RUNX1/3, GATA3 and MYB (Supplementary Table 8). Of 14 CHID1 promoter mutations 12 clustered within a $12 \mathrm{bp}$ region $1 \mathrm{~kb}$ upstream of the TSS and within an AGO1 binding site, corresponding RNAseq was consistent with variants reducing CHID1 expression (Fig. 4b).

To search for significantly mutated cis-regulatory elements (CREs) we restricted our analysis to sequences interacting with promoters through chromatin looping in naïve B-cells [35]. We observed no CREs possessing an excess of mutations and associated with the expression of interacting genes. Additionally we found no evidence of recurrent mutations within UTRs or noncoding RNAs, imposing a threshold of at least five affected tumours.

\section{Mutated pathways}

We next assessed the subtype specificity of driver variation. Additional to documented enrichment of NRAS/KRAS mutations in hyperdiploid ALL and TP53 mutations in hypodiploid/near haploid 
a

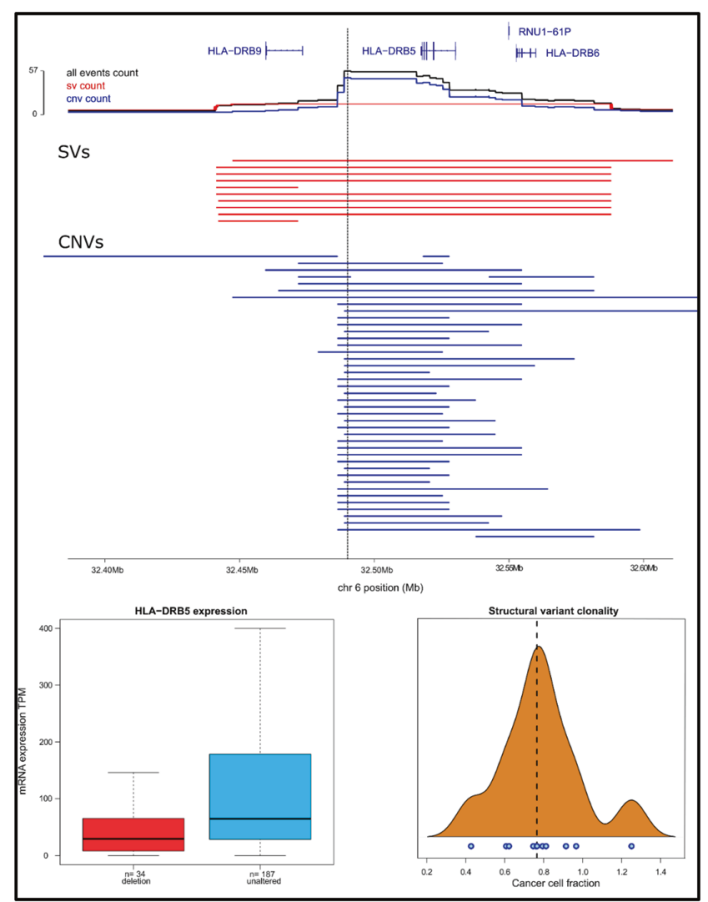

b

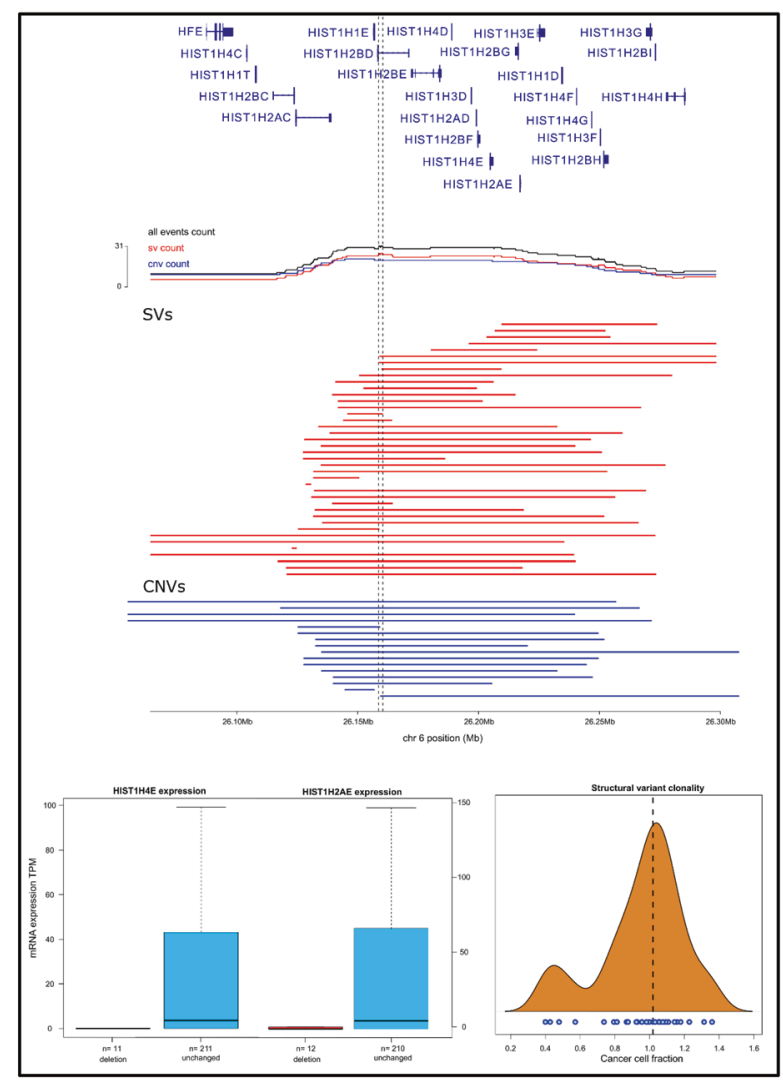

Fig. 3 Recurrent copy number and structural variants. Significantly amplified or deleted regions in CNV data, were filtered retaining only those with an enrichment of structural variants, based on a permutation test. Regional genetic plots showing recurrent deletions mapping to (a) HLA-DRB5 and (b) histone gene cluster 1. Upper panes shows gene position. Line plots show number of tumours with an overlapping variant; blue-CNVs, red-SVs, black - total count (tumours with both SVs and CNVs counted once). Central pane shows the individual variants. For convenience only variants starting or ending in the field of view are plotted. Vertical black lines denote region with the highest deletion frequency. Lower left pane, box plots of gene expression split by mutational status. Lower right pane, density plots of structural variant clonality; blue circles individual SVs. Genomic coordinates from GRCh38.
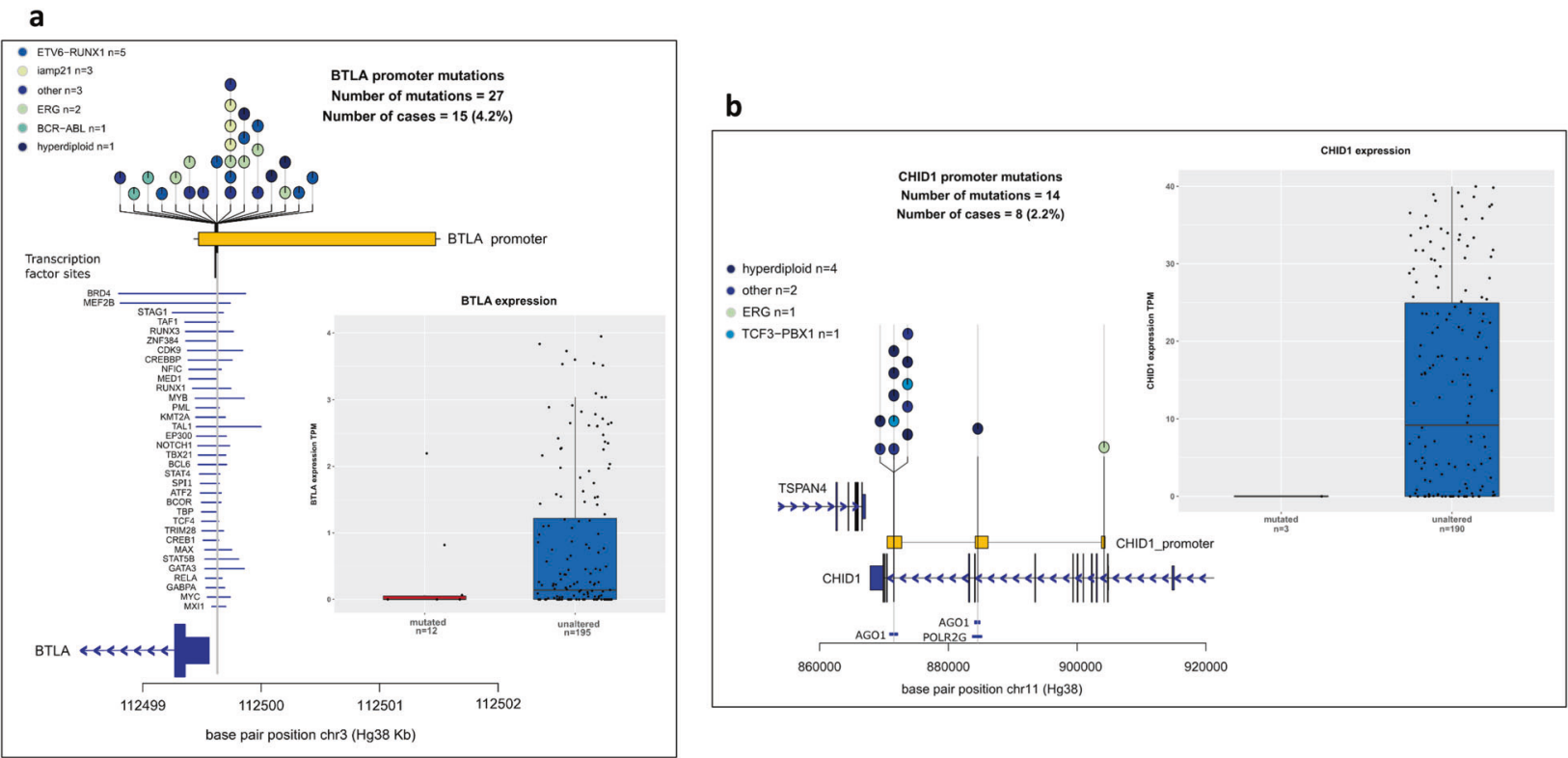

Fig. 4 Non-coding driver mutations. Mutation burden within promoters and their transcriptional impact. Promoter mutations of (a) $B T L A$ and (b) CHID1. Regional plot of mutations (coloured circles) relative to coding sequence (dark blue boxes) and promoter (yellow horizontal bar). Transcription factor binding sites (light blue horizontal line) overlapping mutations were extracted from Encode and ChIP atlas. Grey boxes correspond to transcriptional impact on respective gene. Box and whiskers plot, tumours are split by mutational status, dots represent individual tumours. 


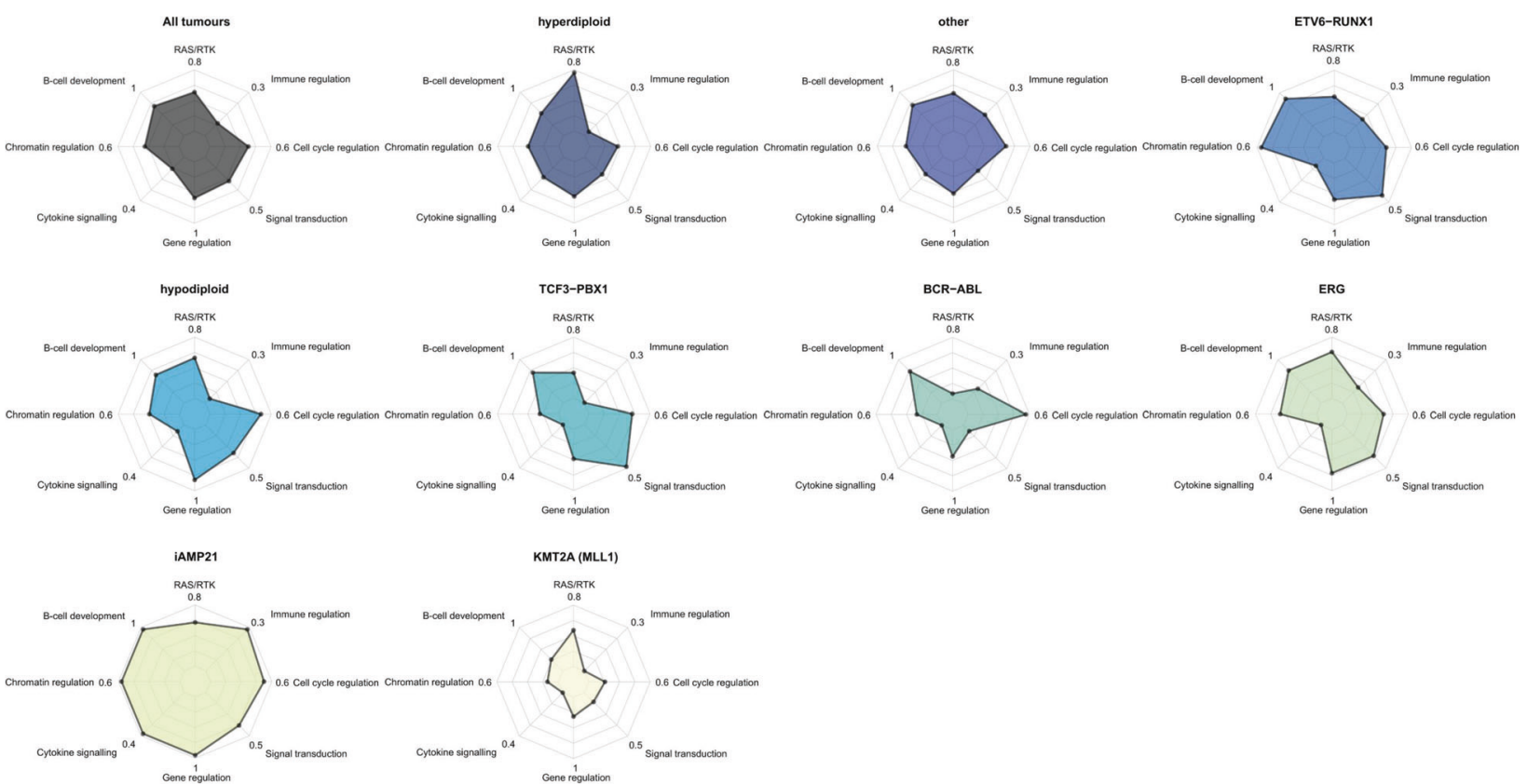

Fig. 5 Pathway analysis and signature analysis. Radar plots showing the most frequently altered pathways for each subtype. Driver genes grouped according to the biological pathway. Somatic alterations for a selected ALL driver genes was compiled from SNV, indel, CNV, SV, RNAseq, and LOH data (CNVs include only focal events). Subtype defining events are excluded (e.g, disruption of ETV6 or RUNX1 in ETV6RUNX1 positive tumours). The proportion of tumours with an alteration in any gene assigned to that pathway is plotted on the radial axis. Each axis is scaled separately. Gene-pathway assignments: RAS/RTK; NRAS, KRAS, PTPN11, FLT3, NF1, ABL1. B-cell development; PAX5, IKZF1, ETV6, ZEB2, RUNX1, TCF3, RAG1, RAG2, EBF1. Chromatin regulation; SETD2, HDAC7, NSD2, CTCF, KMT2A, STAG2, histone gene cluster 1. Cytokine signalling; JAK2, IL7R, CRLF2. Gene regulation; CREBBP, MLLT1, MLLT3, AFF1, BTG1, ERG, TCF4, NCOA6. Signal transduction; TBL1XR1, TBL1X, PBX1, $P A G 1$. Cell cycle regulation; CDKN2A, CDKN2B, RB1. Immune regulation; BTLA, HLA-DRB5.

ALL, we identified a number of other associations (Supplementary Table 9). Notably, TBL1XR1 and ZEB2 mutations were enriched in ERG-deleted ALL (present in $21 \%$ and $14 \%$ of tumours, respectively). iAMP21 tumours were characterised by excess $R B 1$ deletion (40\%) and IL7R mutation (20\%). NF1 mutations were largely confined to near haploid tumours occurring in $45 \%$. Finally, ETV6RUNX1 positive tumours were associated with enrichment for TBLXR1 and RAG1/RAG2 deletions.

Given the identification of deletions within the histone gene cluster 1 and the previous identification of CTCF as a potential ALL driver we explored the transcriptional impact of these lesions, analysing differential expression. We divided tumours according to CTCF (mutated/deleted) and histone 1 cluster (deleted) status, excluding tumours variant for both. We identified five differentially expressed genes in both sets of mutated tumours $\left(P_{\text {Binomial }}=1.5 \times\right.$ $10^{-8}$ ), including CLIC5 and IGF2BPI (Supplementary Table 10 and Supplementary Fig. 11). CLIC5 and IGF2BP1 have been identified as markers of hyperdiploid ALL [55], however, none of the test tumours used in this analysis were hyperdiploid. In total 60 tumours (17\%) harboured alterations (deletions or mutations) in either CTCF or the histone gene cluster 1.

To produce a composite picture of somatic events we clustered drivers by biological pathways (Fig. 5, Supplementary Tables 2 and 11). Alterations of B-cell development genes, were the most frequent, found $70 \%$ of tumours. This analysis confirmed the importance of RAS/RTK alterations in hyperdiploid biology and highlighted a number of other key pathways. Secondary alterations affecting cytokine signalling occurred in 37\% iAMP21 of tumours $\left(Q_{\text {Binomial }}=2.2 \times 10^{-3}\right)$ involving IL7R, JAK2 or CRLF2 (including $3 / 5$ cases of P2RY8-CRLF2 translocation). Alteration of chromatin regulating genes occurred in $56 \%$ of ETV6-RUNX1 positive tumours $\left(Q_{\text {Binomial }}=1.9 \times 10^{-4}\right)$. Hypodiploid tumours were typified by disruption of transcriptional regulators $(Q$ Binomial $=0.012)$, while $T C F 3-P B X 1$ tumours were overrepresented in disruption to genes regulating signal transduction $\left(Q_{\text {Binomial }}=0.012\right)$.
We assessed driver gene mutation clonality, finding most occur both the clonally and sub-clonally (Fig. 6a and Supplementary Fig. 12). An exception was $Z E B 2$ where mutations were always clonal, moreover, mutations of B-cell development and haematopoiesis genes (IKZF1, PAX5 and ZEB2) tended to be clonal. Conversely the majority of RAS/RTK gene mutations were subclonal $(65 \%$; $\left.P_{\text {Fisher }}=0.001\right)$. This was especially true of ERG-deleted tumours where $44 \%$ possessed a subclonal RAS/RTK variant (accounting for $89 \%$ of RAS/RTK mutations in the subtype) compared to $8 \%$ with a clonal variant. Conversely RAS/RTK mutations in hyperdiploid tumours were usually clonal $(60 \%)$, occurring in $44 \%$ of tumours compared to $20 \%$ with only a subclonal variant.

\section{Mutational signatures}

To examine factors promoting tumorigenesis we extracted COSMIC single base signatures (SBS) using SigProfilerExtractor [28]. Ten signatures contributed $>1 \%$ of mutations (Supplementary Fig. 13). SBS5 (aetiology unknown but clock-like) accounted for the most mutations (40\%) and was seen in all tumours (Supplementary Figs. 14 and 15). SBS2 and SBS13 (AID/APOBEC) were almost exclusively confined to ETV-RUNX1 tumours $\left(Q_{\text {Man-Whitney }}=2.3 \times 10^{-33}\right.$ and $Q_{\text {Man-Whitney }}=1.1 \times 10^{-36}$ respectively), whilst SBS7a (UV exposure) was highly enriched in iAMP21 tumours $\left(Q_{\text {Man-Whitney }}=5.3 \times 10^{-12}\right)$ (Supplementary Figs. 16 and 17). SBS7a was associated with the highest mutation rate, 10-fold higher than SBS1 (Supplementary Fig. 18) and was largely responsible for the increased mutation rate in iAMP21 tumours (Supplementary Fig. 19).

SVs in ETV6-RUNX1 positive tumours bear the hallmarks for RAG1 and RAG2 activity [56]. We searched for recurrent DNA motifs at SV breakpoints, firstly agnostically by motif enrichment using HOMER [46], and secondly by assessing the similarity of discovered motifs to the binding sites of candidate mutagenic drivers (Supplementary Table 4). Cohort wide, the most enriched sequences were the RAG heptamer $\left(P<1 \times 10^{-200}\right)$, RAG nonamer 

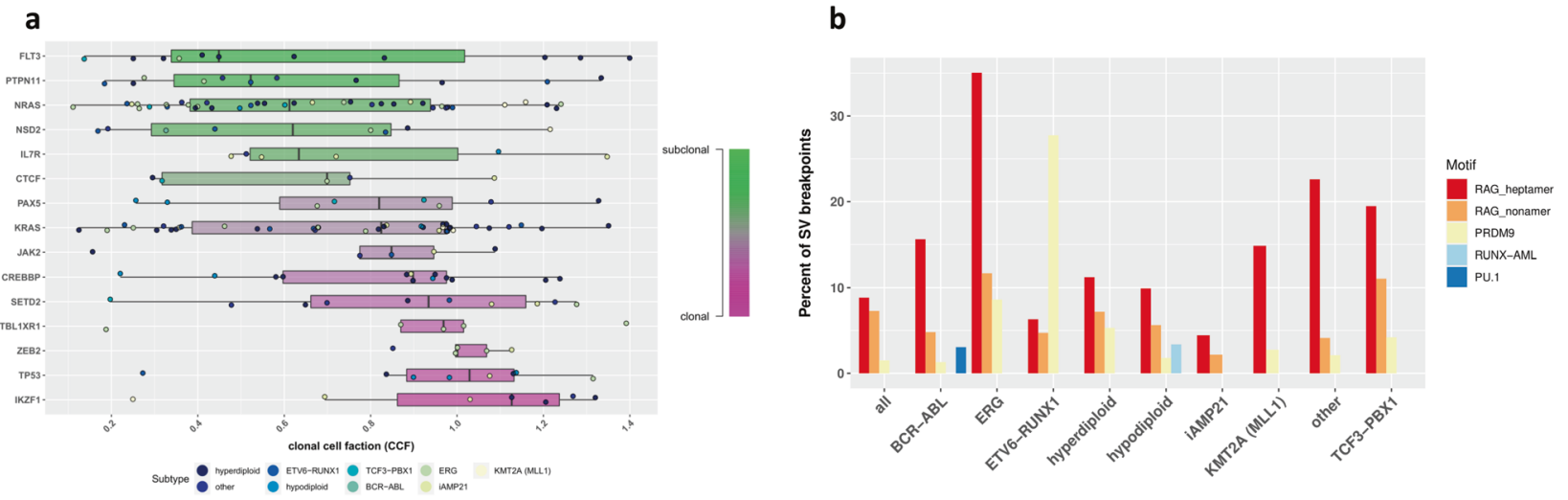

Fig. 6 Driver clonality and SV breakpoint enrichment. a Driver gene mutation (SNV/indels) clonality. Box and whiskers plot showing the proportion clonal mutations for ALL driver genes. Each circle represents a mutation, coloured according to disease subtype, for tumours with multiple mutations in the same gene the variant with the highest clonal cell fraction is retained. b Structural variant motif enrichment. Bar chart showing motif enrichment at SV breakpoints. Two $100 \mathrm{bp}$ of sequences flaking each breakpoint of an SV were extracted and analysed using HOMER. Y-axis; percent of extracted sequences containing motifs.

$\left(P<1 \times 10^{-200}\right)$ and PRDM9 binding motif $\left(P=1 \times 10^{-121}\right)$, found at $8.8,7.2$ and $1.5 \%$ of breakpoints respectively. With the exception of ETV6-RUNX1 positive tumours the most frequent enriched motifs were the RAG heptamer and RAG nonamer, however in ETV6-RUNX1 the most common was the PRDM9 binding motif contained in $28 \%$ of breakpoints $\left(P=1 \times 10^{-162}\right)$ (Fig. 6b). Overall RAG heptamers were observed in both breakpoints of $3 \%$ of SVs.

We also sought evidence of activation-induced deaminase (AID) activity at SV breakpoints. Due to the degenerate nature of AID motifs we used the number of repeats of core AID recognition sequences (Supplementary Table 4) as a proxy of activity. After comparing SVs in immunoglobulin regions we established a cut-off of $>10$ repeats as indicative of AID activity (Supplementary Fig. 20). AID signatures were detected in the breakpoints of $2 \%$ of all SVs, but $17 \%$ of SVs in TCF3-PBX1 positive tumours $\left(P_{\text {Fisher }}=8 \times 10^{-9}\right)$ (Supplementary Fig. 21).

\section{Clonal architecture}

The presence of subclonal populations in tumours was almost universal (observed in $98 \%$ of tumours; Fig. 7a). Most commonly tumours possessed two subclones, however, ERG-deleted tumours tended to have a higher number $\left(Q_{\text {Mann-Whitney }}=0.008\right)$ and KMT2A translocated lower $\left(Q_{\text {Mann-Whitney }}=0.038\right)$ (Supplementary Fig. 22). The distribution of subclone CCF was similar across subtypes, with the exception of hyperdiploid tumours whose subclones had higher CCFs $\left(Q_{\text {Mann-Whitney }}=0.004\right), 50 \%$ having a subclone with a CCF between 0.7 and 0.8 , compared to $9 \%$ of other tumours (Supplementary Fig. 23).

The diversity of cell populations (i.e. heterogeneity) varied across subtypes, hypodiploid and ERG-deleted tumours were the most heterogeneous (median Simpson index $=0.61$ and 0.62 ; $Q_{\text {Mann-Whitney }}=1.7 \times 10^{-3}, 1.13 \times 10^{-3}$ ), while hyperdiploid tumours exhibited lower heterogeneity (Simpson index $=0.45$; $Q_{\text {Mann-Whitney }}=2.8 \times 10^{-7}$ ) (Fig. 7b).

Accounting for mutational frequency, subclones were enriched in driver mutations $\left(P_{\text {Binomal }}=1.8 \times 10^{-5}\right)$ relative to clonal populations. To examine the processes influencing tumour evolution we enumerated the number of subclones with $(\geq 1)$ driver gene mutations for each subtype, comparing this to the frequency observed in remaining subtypes. ERG-deleted tumour subclones were most likely to possess drivers mutations $\left(35 \% ; Q_{\text {binomal }}=\right.$ $0.0016)$, whereas $B C R-A B L 1$ positive tumour subclones contained the lowest frequency $\left(4 \% ; Q_{B i n o m a l}=0.052\right)$ (Supplementary Fig. 24).

To explore the possible contribution of neutral evolution to tumour heterogeneity we used MOBSTER [52], which models variant distribution under neutral evolutionary processes. MOBSTER called neutral 'tails' in the majority of tumours, fitting a median of $12 \%$ (SNVs) and 16\% (SNVs and indels) of variants (Supplementary Fig. 25). Using dNdSCV we found evidence of positive selection in neutral tail compartments which were enriched in NRAS $\left(Q=3.4 \times 10^{-8}\right)$ and $\operatorname{KRAS}\left(Q=1.9 \times 10^{-3}\right)$ mutations. Additionally, rates of non-synonymous substitution in NRAS, KRAS, FLT3, NSD2 were higher in tail compartments than clonal compartments (Supplementary Fig. 26).

\section{DISCUSSION}

By analysing whole genome sequencing and transcriptome data from a large series of ALL patients, we provide for an enhanced understanding of ALL subtype genetics identifying novel candidate coding, noncoding and copy number drivers. Our analysis reveals differences in the mutational and biological pathways processes influencing the initiation and progression of the disease. We also provide evidence of the ongoing selection of subclonal mutations as a ubiquitous feature of ALL evolution.

Around half of ETV6-RUNX1 and iAMP21 tumours are characterised both by an increased mutation rate and enrichment for specific COSMIC single base signatures. AID/APOBEC related signatures, SBS2 and SBS13, were confined to ETV6RUNX1 ALL, while UV-associated SBS7a was highly enriched in IAMP21 positive tumours. SBS7a has previously been reported to occur in ALL tumours at a similar rate [28]. Moreover, SBS7a occurs at similar rates in a number of tumours types lacking UV exposure [28]. These observations provide evidence for an additional mechanistic basis for SBS7a thus implicating unknown germline genetic or environmental factors promoting tumourigenesis of iAMP21 tumours.

We identified three genes enriched in short nucleotide variants, USP8, BSN and SLC35G5. The presence of known sequencing artefacts in USP8 and SLC35G5 necessitates further validation to establish the candidacy of these genes as drivers. $B S N$ is predominantly expressed in neurons where it regulates the release of neurotransmitters consistent with these findings being coincidental.

Deletions of the gene encoding B and T-lymphocyte attenuator (BTLA) have previously been reported in ALL [57], which typically overlap CD200, however, the specific functional mediator has yet to be elucidated. The existence of BTLA promoter SNVs are consistent with this gene, as opposed to CD200, being the driver gene at the $3 q 12.2$ region. 

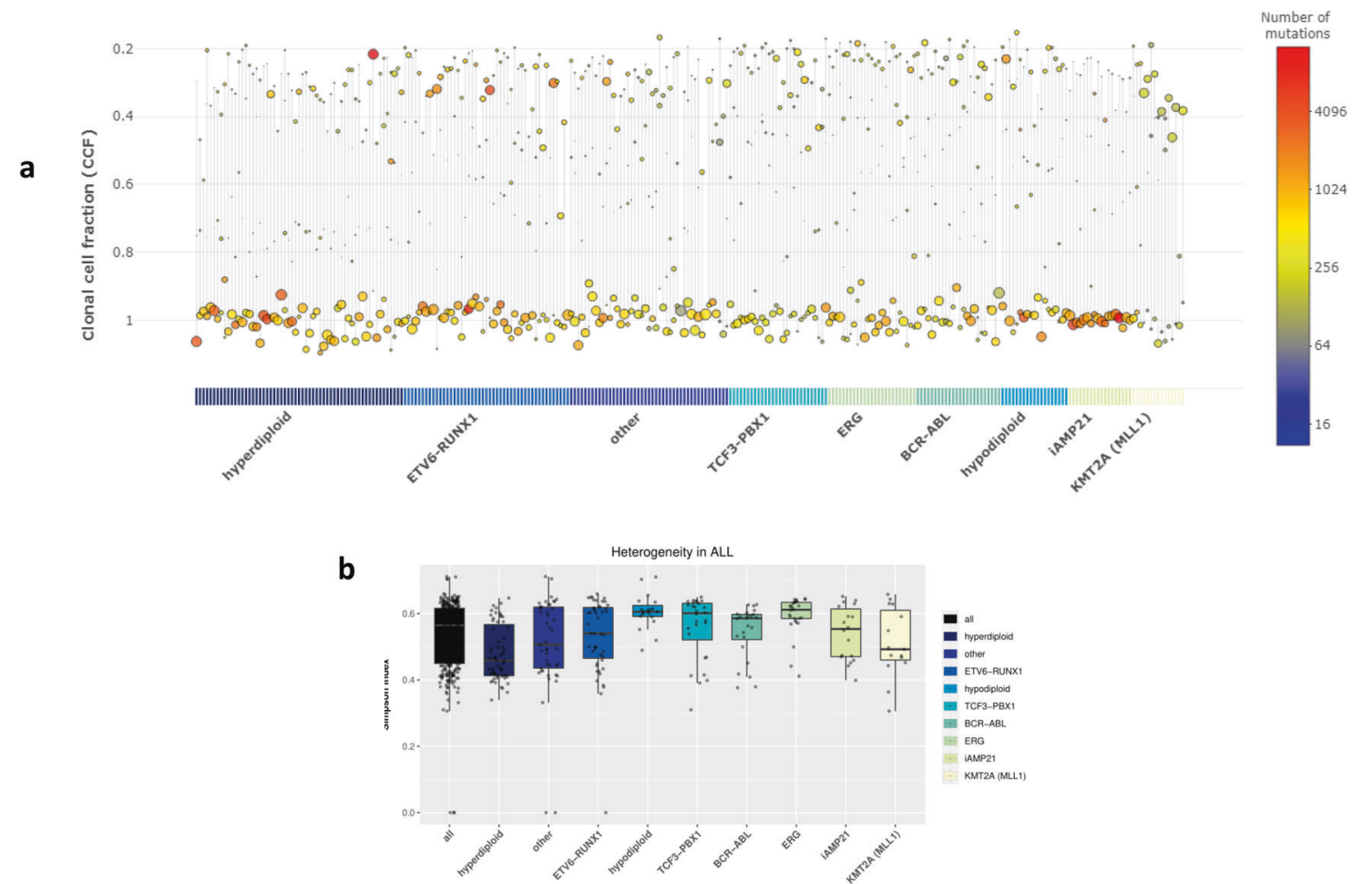

Fig. 7 Clonal architecture and evolution. Variant cancer cell fractions (CCF) were calculated and variants clustered into clonal and subclonal populations. a Distribution of clones. Horizontal lines represent single tumours, circles represent clones; the size and colour of circles corresponding the proportion and number of variants assigned to each clone. Y-axis; clonal frequency (proportion of cell cells with a variant (s)). b Heterogeneity between subtypes. Box and whiskers plot of Simpson index (higher values indicative of increased heterogeneity). Each dot corresponds to a tumour.

We additionally report recurrent copy and structural variation impacting HLA-DRB5. Although the selective basis of these lesions remains unclear, genome-wide association studies in chronic lymphocytic leukaemia [58] and lymphoma [59] have identified germline variants in HLA-DRB5 influencing disease risk.

Around $10 \%$ of tumours possessed a deletion overlapping the histone gene cluster 1, which contains 16 different histone isoforms, including at least two of each core histone. Recurrent histone $\mathrm{H} 1$ mutations have also been reported in around $30 \%-$ $50 \%$ of lymphomas altering chromatin architecture and inducing stem cell-like transcriptional profiles [60]. The further functional characterisation will be required in order to determine the functional gene(s) within these lesions. We show that tumours harbouring histone gene cluster 1 deletions and CTCF alterations share a common transcriptional profile, both downregulating IGF2BP1 and CLIC5. Interestingly, these genes were recently identified alongside CTCF as markers hyperdiploid tumours [55]. No hyperdiploid tumours were included in this analysis, precluding a co-variant effect driving this relationship. Mutations in these genes were however enriched in tumours with no assigned subtype. Alteration of either CTCF or histone gene cluster 1 was common, occurring in 17\% of tumours. Collectively these data raise the possibility of a 'hyperdiploidlike' subtype of ALL.

While there is commonality in disruption of pathways between ALL subtypes there are clear distinctions, not only in the particular biological pathways harbouring mutations but also the clonal distribution of these mutations. These differences have implications for choice of potential targeted therapies and determining which patients will benefit most from their use. As targeting activated oncogenes is generally more tractable than tumour suppressors the biological pathways of most relevance for ALL are RAS/RTK and IL7 signalling. Importantly, RAS/RTK mutations in hyperdiploid tumours were typically clonal, whereas in ERG-deleted ALL mutations were almost exclusively subclonal, suggesting the efficacy of RAS/RTK inhibitors will differ between subtypes. Alteration of IL7 signalling was common in iAMP21 tumours suggesting that JAK2 inhibitors may have utility in this group.

Somatic variants identified as neutrally occurring by MOBSTER were enriched in ALL drivers, indicating that neutral evolution is not a major contributor to genetic heterogeneity in ALL, this may be reflective of the low mutation rate of the disease comparative to most solid cancers. We show that subclonality in ALL is common suggesting Darwinian evolution drives the selection and expansion of mutations and subclones. Consequently, the use of novel targeted therapies should take account of the clonality and heterogeneity of tumours.

\section{Web resources}

Repetitive genomic loci used for variant filtering were downloaded from hgdownload.cse.ucsc.edu/goldenpath/hg38/database/simpleRepeat.txt.gz.

Replication timing was downloaded from 2.replicationdomain. $\mathrm{com} /$.

Smooth the wrapper for structural variant caller Lumpy is available from github.com/brentp/smoove.

Structural variant positional filtering was based on https://github. com/dellytools/delly/blob/master/excludeTemplates/human.hg38. excl.tsv.

Fusionlnspector the RNAseq fusion gene detection software is available from github.com/Fusionlnspector.

Control RNAseq data for GETex and HPA were downloaded from ebi.ac.uk/arrayexpress/experiments/E-GEUV-1/samples/ and $\mathrm{ftp} / / / \mathrm{ftp}$. sra.ebi.ac.uk/vol1/run/ERR315.

Promoters were defined using genode v30 downloaded from ftp:// ftp.ebi.ac.uk/pub/databases /gencode/Gencode_human/release_30/ gencode.v30.annotation.gtf.gz. 
VEGAN package for calculating population diversity is available from github.com/vegandevs/vegan.

HMMcopy is available from http://www.bioconductor.org/ packages/release/bioc/manuals/HMMcopy/man/HMMcopy.pdf

\section{DATA AVAILABILITY}

Data available from DNAnexus (DNAnexus.com) subject to application from St Jude hospital.

\section{CODE AVAILABILITY}

Any unpublished/additional code is available on request from the author

\section{REFERENCES}

1. Inaba H, Greaves $M$, Mullighan CG. Acute lymphoblastic leukaemia. Lancet. 2013;381:1943-55.

2. Harrison CJ. Blood Spotlight on iAMP21 acute lymphoblastic leukemia (ALL), a high-risk pediatric disease. Blood. 2015;125:1383-6.

3. Zhang J, McCastlain K, Yoshihara H, Xu B, Chang $Y$, Churchman ML, et al. Deregulation of DUX4 and ERG in acute lymphoblastic leukemia. Nat Genet 2016;48:1481-9.

4. Carroll WL. Safety in numbers: hyperdiploidy and prognosis [Internet]. Blood. 2013;48:2374-6.

5. Moorman AV, Richards SM, Robinson HM, Strefford JC, Gibson BES, Kinsey SE, et al. Brief report: prognosis of children with acute lymphoblastic leukemia (ALL) and intrachromosomal amplification of chromosome 21 (iAMP21). Blood. 2007;109:2327-30.

6. Steeghs EMP, Boer JM, Hoogkamer AQ, Boeree A, de Haas V, de Groot-Kruseman $\mathrm{HA}$, et al. Copy number alterations in B-cell development genes, drug resistance, and clinical outcome in pediatric B-cell precursor acute lymphoblastic leukemia. Sci Rep. 2019;9:1-11.

7. Paulsson K, Johansson B. High hyperdiploid childhood acute lymphoblastic leukemia. Genes Chromosomes Cancer. 2009;48:637-60.

8. Pui $\mathrm{CH}$, Evans WE. A 50-year journey to cure childhood acute lymphoblastic leukemia. Semin Hematol. 2013;50:185-96.

9. Freyer DR, Devidas M, La M, Carroll WL, Gaynon PS, Hunger SP, et al. Postrelapse survival in childhood acute lymphoblastic leukemia is independent of initial treatment intensity: A report from the Children's Oncology Group. Blood. 2011;117:3010-5.

10. Nguyen K, Devidas M, Cheng SC, La M, Raetz EA, Carroll WL, et al. Factors influencing survival after relapse from acute lymphoblastic leukemia: A Children's Oncology Group study. Leukemia. 2008;22:2142-50.

11. Amgen slaps record-breaking $\$ 178 \mathrm{~K}$ price on rare leukemia drug Blincyto. https:// www.fiercepharma.com/marketing/amgen-slaps-record-breaking-178k-price-onrare-leukemia-drug-blincyto. Retrieved 25/10/2021.

12. Paulsson K, Horvat A, Strömbeck B, Nilsson F, Heldrup J, Behrendtz $M$, et al. Mutations of FLT3, NRAS, KRAS, and PTPN11 are frequent and possibly mutually exclusive in high hyperdiploid childhood acute lymphoblastic leukemia. Genes Chromosom Cancer. 2008:47:26-33.

13. Ding LW, Sun QY, Tan KT, Chien W, Thippeswamy AM, Yeoh AEJ, et al. Mutational landscape of pediatric acute lymphoblastic leukemia. Cancer Res. 2017;77:390-400

14. Paulsson K, Lilljebjörn $H$, Biloglav A, Olsson L, Rissler M, Castor A, et al. The genomic landscape of high hyperdiploid childhood acute lymphoblastic leukemia. Nat Genet. 2015;47:672-7.

15. Mullighan CG. The genomic landscape of acute lymphoblastic leukemia in children and young adults. Hematol (United States). 2014;2014:174-80.

16. Mullighan CG, Goorha S, Radtke I, Miller CB, Coustan-Smith E, Dalton JD, et al. Genome-wide analysis of genetic alterations in acute lymphoblastic leukaemia. Nature. 2007;446:758-64.

17. Liu YF, Wang BY, Zhang WN, Huang JY, Li BS, Zhang M, et al. Genomic profiling of adult and pediatric B-cell acute lymphoblastic leukemia. EBioMedicine. 2016;8:173-83.

18. Li H, Durbin R. Fast and accurate short read alignment with Burrows-Wheeler transform. Bioinformatics. 2009;25:1754-60.

19. Dobin A, Davis CA, Schlesinger F, Drenkow J, Zaleski C, Jha S, et al. STAR: ultrafast universal RNA-seq aligner. Bioinformatics. 2013;29:15-21.

20. Li B, Dewey CN. RSEM: Accurate transcript quantification from RNA-Seq data with or without a reference genome. BMC Bioinformatics. 2011;12:323.

21. Zhang Y, Parmigiani G, Johnson WE ComBat-Seq: batch effect adjustment for RNA-Seq count data. bioRxiv. 2020;2020.01.13.904730.
22. Haas BJ, Dobin A, Li B, Stransky N, Pochet N, Regev A. Accuracy assessment of fusion transcript detection via read-mapping and de novo fusion transcript assembly-based methods. Genome Biol. 2019;20:213.

23. Ardlie KG, DeLuca DS, Segrè AV, Sullivan TJ, Young TR, Gelfand ET, et al. The Genotype-Tissue Expression (GTEx) pilot analysis: Multitissue gene regulation in humans. Science (80-). 2015;348:648-60.

24. Uhlén M, Fagerberg L, Hallström BM, Lindskog C, Oksvold P, Mardinoglu A, et al. Tissue-based map of the human proteome. Science (80-). 2015;23:347.

25. Love MI, Huber W, Anders S. Moderated estimation of fold change and dispersion for RNA-seq data with DESeq2. Genome Biol. 2014;15:550.

26. Kim S, Scheffler K, Halpern AL, Bekritsky MA, Noh E, Källberg M, et al. Strelka2: fast and accurate calling of germline and somatic variants. Nat Methods. 2018;15:591-4.

27. Mayakonda A, Lin DC, Assenov Y, Plass C, Koeffler HP. Maftools: efficient and comprehensive analysis of somatic variants in cancer. Genome Res. 2018;28:1747-56.

28. Alexandrov LB, Kim J, Haradhvala NJ, Huang MN, Tian Ng AW, Wu Y, et al. The repertoire of mutational signatures in human cancer. Nature. 2020;578:94-101.

29. Lawrence MS, Stojanov P, Mermel CH, Robinson JT, Garraway LA, Golub TR, et al. Discovery and saturation analysis of cancer genes across 21 tumour types. Nature. 2014;505:495-501.

30. Martincorena I, Raine KM, Gerstung M, Dawson KJ, Haase K, Van Loo $P$, et al. Universal patterns of selection in cancer and somatic tissues. Cell. 2017;171:1029-41.e21.

31. Mularoni L, Sabarinathan R, Deu-Pons J, Gonzalez-Perez A, López-Bigas N. OncodriveFML: a general framework to identify coding and non-coding regions with cancer driver mutations. Genome Biol. 2016;17:128.

32. Wilson DJ. The harmonic mean p-value for combining dependent tests. Proc Natl Acad Sci U S A. 2019;116:1195-200.

33. McLaren W, Gil L, Hunt SE, Riat HS, Ritchie GRS, Thormann A, et al. The ensembl variant effect predictor. Genome Biol. 2016;17:122.

34. Orlando G, Law PJ, Cornish AJ, Dobbins SE, Chubb D, Broderick P, et al. Promoter capture $\mathrm{Hi}-\mathrm{C}$-based identification of recurrent noncoding mutations in colorectal cancer [Internet]. Nat Genet. 2018;50:1375-80.

35. Javierre BM, Sewitz S, Cairns J, Wingett SW, Várnai C, Thiecke MJ, et al. Lineagespecific genome architecture links enhancers and non-coding disease variants to target gene promoters. Cell. 2016;167:1369-84.e19.

36. Melton C, Reuter JA, Spacek DV, Snyder M. Recurrent somatic mutations in regulatory regions of human cancer genomes. Nat Genet. 2015;47:710-6.

37. Talevich E, Shain AH, Botton T, Bastian BC. CNVkit: Genome-Wide Copy Number Detection and Visualization from Targeted DNA Sequencing. PLoS Comput Biol. 2016;12:e1004873.

38. Nik-Zainal S, Van Loo P, Wedge DC, Alexandrov LB, Greenman CD, Lau KW, et al. The life history of 21 breast cancers. Cell. 2015;162:924.

39. Mermel CH, Schumacher SE, Hill B, Meyerson ML, Beroukhim R, Getz G. GISTIC2.0 facilitates sensitive and confident localization of the targets of focal somatic copy-number alteration in human cancers. Genome Biol. 2011;12:R41.

40. Chen X, Schulz-Trieglaff $O$, Shaw R, Barnes B, Schlesinger F, Källberg $M$, et al. Manta: Rapid detection of structural variants and indels for germline and cancer sequencing applications. Bioinformatics. 2016;32:1220-2.

41. Layer RM, Chiang C, Quinlan AR, Hall IM. LUMPY: a probabilistic framework for structural variant discovery. Genome Biol. 2014;15:R84.

42. Rausch T, Zichner T, Schlattl A, Stütz AM, Benes V, Korbel JO. DELLY: structural variant discovery by integrated paired-end and split-read analysis. Bioinformatics. 2012;28:i333-i339.

43. Li Y, Roberts ND, Wala JA, Shapira O, Schumacher SE, Kumar K, et al. Patterns of somatic structural variation in human cancer genomes. Nature. 2020;578:112-21.

44. Cmero M, Ong CS, Yuan K, Schröder J, Mo K, Group PE and HW, et al. SVclone: inferring structural variant cancer cell fraction. bioRxiv. 2017;4:172486.

45. Cortés-Ciriano I, Lee JJK, Xi R, Jain D, Jung YL, Yang L, et al. Comprehensive analysis of chromothripsis in 2,658 human cancers using whole-genome sequencing. Nat Genet. 2020;52:331-41.

46. Heinz S, Benner C, Spann N, Bertolino E, Lin YC, Laslo P, et al. Simple combinations of lineage-determining transcription factors Prime cis-regulatory elements required for macrophage and $B$ cell identities. Mol Cell. 2010;38:576-89.

47. Sandelin A, Alkema W, Engström P, Wasserman WW, Lenhard B. JASPAR: an openaccess database for eukaryotic transcription factor binding profiles. Nucleic Acids Res. 2004;1:32.

48. Harrison CJ, Haas O, Harbott J, Biondi A, Stanulla M, Trka J, et al. Detection of prognostically relevant genetic abnormalities in childhood B-cell precursor acute lymphoblastic leukaemia: recommendations from the Biology and Diagnosis Committee of the International Berlin-Frankfürt-Münster study group. Br J Haematol. 2010;151:132-42. 
10

49. Howie BN, Donnelly P, Marchini J. A flexible and accurate genotype imputation method for the next generation of genome-wide association studies. PLoS Genet. 2009;5:e1000529.

50. Yuan K, Macintyre G, Liu W, Markowetz F. Ccube: a fast and robust method for estimating cancer cell fractions. bioRxiv. 2018;484402.

51. Dixon P. VEGAN, a package of R functions for community ecology [Internet]. J Veg Sci. 2003;14:927-30.

52. Caravagna G, Heide T, Williams MJ, Zapata L, Nichol D, Chkhaidze K, et al. Subclonal reconstruction of tumors by using machine learning and population genetics. Nat Genet. 2020;52:898-907.

53. Tran TH, Hunger SP. The genomic landscape of pediatric acute lymphoblastic leukemia and precision medicine opportunities. Sem Cancer Biol. 2020;107: 2411-2502.

54. Lai Daniel, Ha Gavin SS. HMMcopy: copy number prediction with correction for GC and mappability bias for HTS data. 2020.

55. Yang $M$, Vesterlund $M$, Siavelis I, Moura-Castro LH, Castor A, Fioretos $T$, et al. Proteogenomics and $\mathrm{Hi}-\mathrm{C}$ reveal transcriptional dysregulation in high hyperdiploid childhood acute lymphoblastic leukemia. Nat Commun. 2019;1:10.

56. Papaemmanuil E, Rapado I, Li Y, Potter NE, Wedge DC, Tubio J, et al. RAGmediated recombination is the predominant driver of oncogenic rearrangement in ETV6-RUNX1 acute lymphoblastic leukemia. Nat Genet. 2014;46:116-25.

57. Ghazavi F, Clappier E, Lammens T, Suciu S, Caye A, Zegrari S, et al. CD200/BTLA deletions in pediatric precursor B-cell acute lymphoblastic leukemia treated according to the EORTC-CLG 58951 protocol. Haematologica. 2015;100:1311-9.

58. Slager SL, Rabe KG, Achenbach SJ, Vachon CM, Goldin LR, Strom SS, et al. Genome-wide association study identifies a novel susceptibility locus at $6 \mathrm{p} 21.3$ among familial CLL. Blood. 2011;117:1911-6.

59. Conde L, Halperin E, Akers NK, Brown KM, Smedby KE, Rothman N, et al. Genomewide association study of follicular lymphoma identifies a risk locus at 6 p21.32. Nat Genet. 2010;42:661-4.

60. Yusufova N, Kloetgen A, Teater M, Osunsade A, Camarillo JM, Chin CR, et al. Histone $\mathrm{H} 1$ loss drives lymphoma by disrupting 3D chromatin architecture. Nature. 2021;589:299-305.

\section{ACKNOWLEDGEMENTS}

This work was supported by Cancer Research UK (C1298/A8362) and Blood Cancer UK.

\section{AUTHOR CONTRIBUTIONS}

J.S., A.C., P.L. performed bioinformatic and statistical analyses. P.H., A.C. and B.K. provided additional bioinformatics support. J.S. and R.H. drafted the manuscript.

\section{COMPETING INTERESTS}

The authors declare no competing interests.

\section{ADDITIONAL INFORMATION}

Supplementary information The online version contains supplementary material available at https://doi.org/10.1038/s41408-021-00570-9.

Correspondence and requests for materials should be addressed to James B. Studd.

Reprints and permission information is available at http://www.nature.com/ reprints

Publisher's note Springer Nature remains neutral with regard to jurisdictional claims in published maps and institutional affiliations.

(i) Open Access This article is licensed under a Creative Commons Attribution 4.0 International License, which permits use, sharing, adaptation, distribution and reproduction in any medium or format, as long as you give appropriate credit to the original author(s) and the source, provide a link to the Creative Commons license, and indicate if changes were made. The images or other third party material in this article are included in the article's Creative Commons license, unless indicated otherwise in a credit line to the material. If material is not included in the article's Creative Commons license and your intended use is not permitted by statutory regulation or exceeds the permitted use, you will need to obtain permission directly from the copyright holder. To view a copy of this license, visit http://creativecommons. org/licenses/by/4.0/.

(c) The Author(s) 2021 